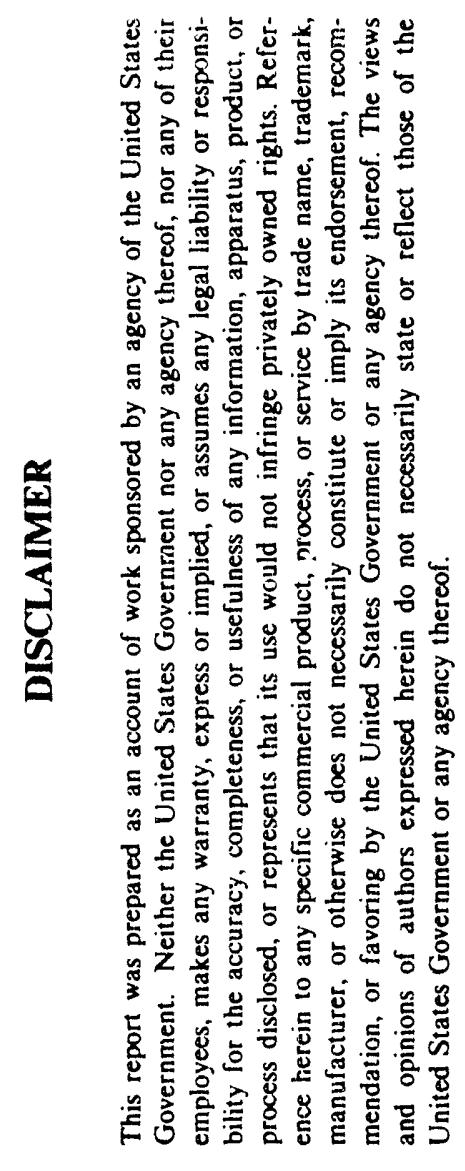

SCHOTTKY BARRIER HEIGHT CONTROL AT EPITAXIAL NiAI/GaAs(001)

INTERFACES BY MEANS OF VARIABLE BANDGAP INTERLAYERS

S. A. Chambers

November 1992

Presented at the American Vacuum Society 39th National Symposium and Topical Conference November 9-13, 1992 Chicago, Illinois

Prepared for the U.S. Department of Energy under Contract DE-ACO6-76RLO 1830

Pacific Northwest Laboratory Richland, Washington 99352 
(To be presented as an invited paper at the 39th National Symposium of the American

Vacuum Society, and submitted to J. Vac. Sci. Technol. A)

\title{
Schottky Barrier Height Control at Epitaxial NiAl/GaAs(001) Interfaces by Means of Variable Bandgap Interlayers
}

\author{
Scott A. Chambers \\ Molecular Science Research Center \\ Pacific Northwest Laboratory* \\ Richland, Washington
}

\begin{abstract}
We discuss recent developments in the use of interlayers to tailor the Schottky barrier height (SBH) at a metal/GaAs interface. The goal has been to gain control of band bending in the interfacial region by modifying both the interface Fermi energy and the charge density in the depletion region. Our approach has been to grow both the interlayer and the metal overlayer under ultrahigh vacuum conditions by molecular beam epitaxy, and then to determine the chemistry of interface formation, structure, and band bending by $x$-ray photoelectron spectroscopy and diffraction and by low-energy electron diffraction. The interface Fermi energy can be changed from the usual midgap value of $0.7-0.8 \mathrm{eV}$ relative to the band edge by the use of epitaxial transition metal aluminide (TMA) overlayers such as NiAl. The unique chemistry of interface formation between this intermetallic compound and $\mathrm{GaAs}$ pins the Fermi level $\sim 0.3-0.4 \mathrm{eV}$ above the valence band maximum, resulting in a SBH of $\sim 1 \mathrm{eV}$. The SBH can be increased to $\sim 1.2 \mathrm{eV}$ by the use of a wide bandgap interlayer such as AlAs. The charge density in the depletion region can be changed by
\end{abstract}

* Pacific Northwest Laboratory is operated for the U.S. Department of Energy by Battelle Memorial Institute under Contract DE-AC06-76 RLO 1830. 
growing an $n^{+}$-type group IV interlayer between the TMA overlayer and GaAs substrate. Charge transfer from the interlayer to an n-type substrate reduces the space charge density, thereby lowering the band bending and, thus, the SBH to $\sim 0.5 \mathrm{eV}$. The use of these interlayers then produces a range of $\mathrm{SBH}$ values of $\sim 0.7 \mathrm{eV}$, which is a significant improvement over the rather narrow range of $0.1-0.2 \mathrm{eV}$ that results from conventional metallizations. We discuss the fundmental interface science that underpins these results, and then conclude with an application to complementary digital GaAs circuit design that may significantly reduce gate leakage.

\section{Introduction}

The tendency of III-V compound semiconductors to exhibit surface and interface Fermi-level pinning near midgap constitutes a significant limitation for several device technologies. ${ }^{1}$ For instance, true Ohmic contacts cannot be fabricated, because Fermi-level pinning near midgap creates an electrostatic barrier that must be traversed by carriers crossing the interface. In the absence of tunneling, such a barrier results in rectifying behavior in the current-voltage (I-V) curves. In order to achieve linear I-V curves characteristic of true Ohmic contacts, device technologists currently utilize a complex metallization scheme that includes $\mathrm{Ge}$ and $\mathrm{Zn}$ for $\mathrm{n}$-type and p-type substrates, respectively. Subsequent alloying of the metallization results in contacts that exhibit nearly linear I-V behavior and possess contact resistances typical of true Ohmic contacts. ${ }^{2}$ It is thought that $\mathrm{Ge}(\mathrm{Zn})$ atoms indiffuse, giving rise to a heavily-doped n-type (p-type) region near the interface. The presence of these excess donors causes the spatial width of the electrostatic barrier to be sufficiently small that carriers can effectively tunnel across the interface. Unfortunately, formation of this Ohmic contact consumes several hundred $\AA$ of substrate material, thereby precluding the fabrication of shallow-junction devices. Furthermore, this metal/III-V interface is rough and chemically inhomogeneous. ${ }^{3}$ A more ideal Ohmic contact would utilize a metallization scheme in which the Fermi level is very close to the 
valence (conduction) band for p-type (n-type) substrates, and for which the interface is more planar and chemically homogeneous.

Similarly, low-leakage-current rectifying contacts are not possible when Fermi-level pinning occurs near midgap. A Schottky barrier height of $\sim 0.7-0.8 \mathrm{eV}$ (a typicai micigap value for GaAs) is not large enough to prevent gate leakage from occurring when the gate is biased to allow source-to-drain conductance. Such leakage results is low noise margins in complementary digital circuit applications, as well as undesirable power dissipation. ${ }^{4}$ Thus, it is desirable to maximize the band bending by moving the Fermi level as far away from midgap as possible.

Both of these technological goals can be realized by gaining control of the interface Fermi energy. A considerable amount of effort has been expended over several decades in attempting to understand the mechanism of Schottky barrier height (SHB) formation. ${ }^{5-7}$ Several models have been proposed, but none has proven to be universally satisfactory in explaining the body of data that now exists. ${ }^{8-14}$ It appears that complex interfacial phenomena result in electronic state distributions in the forbidden gap that are exceedingly difficult to understand, let alone control. In this paper, we discuss the use of variable band gap interlayers that are thin compared to typical electron inelastic scattering mean free paths to achieve control over the interface Fermi level. This approach allows the investigator to utilize the properties of two interfaces rather than one in an attempt to tailor the SBH. We have combined molecular beam epitaxial (MBE) growth techniques with in-situ characterization by $\mathrm{x}$-ray photoelectron spectroscopy (XPS), $\mathrm{x}$-ray photoelectron diffraction (XPD), and low-energy electron diffraction (LEED). This combination of probes allows us to correlate electronic properties with the chemistry and structure of the interfacial region in an attempt to understand the forces that control the SBH when interlayers are used. 


\section{Experimental}

All experiments were carried out in a custom-built ultrahigh vacuum (UHV) system that combines rapid substrate entry with MBE, XPS, XPD, and LEED. The MBE system includes metal and semiconductor evaporation capability from EPI System Knudson cells with cryogenic cooling, and flux determinations by quartz-crystal oscillators and a residual gas analyzer. The XPS and XPD measurements were carried out with a Surface Science Instruments Series 300 x-ray photoelectron spectrometer specially modified to perform scanned-angle XPD measurements under computer control. This spectrometer system utilizes a monochromatic AlK $\alpha$ x-ray source, a hemispherical analyzer with externallyactuated apertures for XPD, and a multichannel detector. Scanned-angle XPD measurements were carried out by rotating the sample about two orthogonal axes while keeping the x-ray source and analyzer stationary.

$\mathrm{GaAs}(001)$ substrates were prepared by three-solvent degreasing, etching in a dilute $\mathrm{H}_{2} \mathrm{SO}_{4} / \mathrm{H}_{2} \mathrm{O}_{2} / \mathrm{H}_{2} \mathrm{O}$ mixture, rinsing in deionized water, and heating in air to form volatile oxides on the surface. Samples were then either entered directly into the system described above for some of the earlier studies, or more recently, into a Perkin Elmer model 430 MBE, a Varian Gen-II MBE, or an Emcore model GS-3200 metal-organic chemical vapor deposition (MOCVD) reactor for buffer layer growth. Samples entered directly into the system described in the previous paragraph were annealed briefly at $\sim 600^{\circ} \mathrm{C}$ either in UHV or in an As4 overpressuce to desorb the surface oxides. The resulting surfaces were clean and ordered, typically exhibiting a (1x1) LEED pattern with some extra spots characteristic of a partial surface reconstruction. The MBE- and MOCVD-grown buffer layers were passivated with $A s_{4}$ and $\mathrm{GaSe}_{\mathrm{x}} \mathrm{As} s_{1-\mathrm{x}}$ overlayers, respectively, to permit through-air transfer to the primary experimental system. Once under UHV conditions, the passivating layers were desorbed at temperatures between $450^{\circ}$ and $550^{\circ} \mathrm{C}$ to expose clean, anionstabilized surfaces with the appropriate surface reconstruction as det rmined by LEED. Specifically, annealing MBE-grown $\mathrm{n}$ - and p-type buffer layers with an As passivation 
layer at $450^{\circ} \mathrm{C}$ produced the $\mathrm{c}(2 \times 8) /(2 \times 4)$ reconstruction that accompanies As dimer formation in the terminal layer with every third dimer pair is missing. Annealing Se-doped buffer layers grown by MOCVD and passivated with $\mathrm{GaSe}_{\mathrm{x}} \mathrm{As}_{1-\mathrm{x}}$ to $450^{\circ} \mathrm{C}$ in $\mathrm{UHV}$ produces an anion-stabilized $(2 \times 1)$ surface in which the outer several layers consist of epitaxial GaSe $\mathrm{As}_{1-\mathrm{x}}$ on GaAs. Here, $\mathrm{x}$ is $\sim 0.8$ in the surface layer and drops to $\sim 0.2$ at the fourth anion layer. This determination was made by XPD, and will be described in more detail in section IIID. This surface is of considerable interest in and of itself in that the surface band bending is very low as a result of the Se passivation, as discussed in section IIID.

\section{Results and Discussion}

\section{A. General considerations}

One must be able to control the band bending and interface Fermi energy in order to tailor the SBH at a metal/semiconductor interface. Three criteria come to mind when using variable bandgap interlayers to achieve this goal. If we first consider maximizing the SBH at a metal/n-semiconductor interface, it is desirable to use an interlayer for which: (i) the bandgap of the interlayer is greater than that of the substrate, (ii) the majority of the band offset between the substrate and the interlayer is in the conduction band, and (iii) the interlayer in combination with the metal induce a large band bending in the substrate. Similarly, in order to minimize the SBH, one would want to utilize an interlayer for which (i) the bandgap of the interlayer is less than that of the substrate, (ii) the majority of the band offset is in the conduction band, and (iii) the interlayer in combination with the metal do not induce a large band bending in the substrate. These conditions are depicted schematically in Figure 1.

As the energy diagrams to the right of Figure 1 show, fulfilling these criteria produces band behavior that is conducive to preventing or facilitating charge transfer across the interface. A wide bandgap interlayer in combination with high band bending $\left(\triangle \mathrm{E}_{\mathrm{CB}}\right.$ $\left.F_{L}\right)$ and a large conduction band offset $\left(\Delta \mathrm{E}_{C}\right)$ enhances the $\mathrm{SBH}\left(\phi_{\mathrm{B}}\right)$ relative to what can 
be achieved on the substrate without such an interlayer. Furthermore, tunneling through the interlayer can be controlled by varying the interlayer thickness. Similarly, a heavilydoped n-type interlayer with a bandgap smaller than that of the substrate will significantly reduce the SBH, particularly if the majority of the band offset is in the conduction band. Fermi-level equilibration between the substrate and the interlayer will lower the band bending, which is also required for a low SBH value. As we shall see in sections IIIC-E, these conditions can be realized by judiciously choosing the bandgap and doping of the interlayer.

\section{B. Epitaxy of $\mathrm{NiAl}$ on $\mathrm{GaAs}(001)$ and the resulting $\mathrm{SBH}$}

Transition metal aluminides (TMA) such as NiAl possess several properties that make them highly desirable as next-generation rectifying metallizations in III-V field-effect transistor (FET) circuit technology. 15 The melting point of stoichiometric NiAl is $1638^{\circ} \mathrm{C}$. Thus, the metal should possess good thermal stability at the elevated temperatures associated with both processing and operation. In addition, the resistivity of $\mathrm{NiAl}$ is 9.8 $\mu \Omega-\mathrm{cm}$, which is only about an order of magnitude higher than that of the noble metals. Thus, the series resistance of the contact is expected to be quite low, which reduces RC time delays in high-frequency applications. $\mathrm{NiAl}$ possesses a $\mathrm{CsCl}$ crystal structure with a lattice constant equal to approximately half that of GaAs. Therefore, the compound can be grown epitaxially on GaAs and other III.V semiconductors with comparably lattice constants. In fact, $\mathrm{NiAl}$ is exactly lattice matched to In $0.30 \mathrm{Ga} 0.70 \mathrm{As}$. The absence of grain boundaries in the metallization should minimize outdiffusion of $\mathrm{Ga}$ and $\mathrm{As}$ atoms during operation at elevated temperature, thus preserving the stoichiometry of the semiconductor layers below the contact. Finally, NiAl/n-GaAs(001) interfaces exhibit a SBH of 0.9-1.0 $\mathrm{eV}$, which is an improvement over the typical midgap values of 0.7-0.8 eV exhibited by virtually all other rectifying metals now being used or under consideration in III-V device technology. As explained above, a higher SBH decreases gate leakage, which is significant advantage in most device technologies. 
Overlayers of $\mathrm{NiAl}$ on $(\mathrm{Ga}, \mathrm{Al}) \mathrm{As}(001)$ as well as on In $0.15 \mathrm{Ga} 0.85 \mathrm{As}$ can be grown epitaxially at temperatures as low as $50^{\circ}-100^{\circ} \mathrm{C} .15$ The crystal symmetries and lattice constants strongly suggest that the orientational relationship between the two materials should be $\{001\}_{\mathrm{NiAl}} \|\{001\}_{\mathrm{GaAs}}$ and $<011>_{\mathrm{NiAl}} \|<011>_{\mathrm{GaAs}}$. Transmission electron micrographs by Sands et al. ${ }^{15}$ as well as our own detailed XPD studies ${ }^{16}$ show that this orientation is assumed, and that epitaxial films of high structural quality do indeed form. However, the upper surface of the NiAl finins exhibit some roughness on an atomic scale, in the form of a high density of single steps. 15,16

Epitaxial overgrowth of $\mathrm{NiAl}$ on $\mathrm{GaAs}(001)$ occurs despite limited reactivity at the interface between metal atoms in the incident flux and the substrate. This reactivity is detected by the presence of extra peaks in the high energy resolution $\mathrm{Ga}$ and $\mathrm{As} 3 \mathrm{~d}$ corelevel XPS spectra. ${ }^{17}$ Such peaks result from the liberation of Ga and As atoms from the substrate, which may be followed by compound or solid solution formation with metal atoms. The spatial distribution of these species can be at least qualitatively inferred from plots of the reduced total $\mathrm{Ga}$ and As $3 \mathrm{~d}$ intensities as a function of overlayer coverage. Such plots are shown in the top panel of Figure 2. Linear behavior indicates that the reacted species remains trapped at the interface, as in the case of $\mathrm{Ga}$. A positive deviation from linearity indicates outdiffusion and possibly surface segregation, as in the case of As. When the reacted species remains trapped at the interface, it is possible to model the ratio of reacted-to-substrate peak intensities as a function of coverage taken from the the highresolution spectra to obtain the approximate number of reacted substrate layers. Such a plot is shown in the bottom panel of Figure 2 for Ga $3 \mathrm{~d}$ emission. Here, $\mathrm{n}$ is the number of GaAs bilayers consumed in the reaction with the incident $\mathrm{Ni}$ and $\mathrm{Al}$. As seen in Figure 2, $\mathrm{n}$ peaks at $\sim 2.5$ by an overlayer coverage of $\sim 10$ monolayers, and then does not further increase with coverage. Studies of epitaxial growth at higher substrate temperatures suggest that the interface extent of reaction is kinetically limited, ${ }^{18}$ and that further consumption of the substrate at $100^{\circ} \mathrm{C}$ is inhibited by the lack of thermal mobility of $\mathrm{Ni}$ 
and/or $\mathrm{Al}$ atoms in the near-surface region. The reaction to form NiAl, which is highly stable thermodynamically, represents a competitive pathway to reaction with the substrate. Indeed, a comparison of $\mathrm{Ga}$ and $\mathrm{As} 3 \mathrm{~d}$ spectra after growth of $\mathrm{Ni}, \mathrm{Al}$, and $\mathrm{NiAl}$ reveals considerably less reaction with the substrate when $\mathrm{Ni}$ and $\mathrm{Al}$ are coevaporated than when $\mathrm{Ni}$ and $\mathrm{Al}$ are evaporated separately. ${ }^{19}$

$\mathrm{SBH}$ values, as determined by XPS, of $1.07 \pm 0.06 \mathrm{eV}$ and $0.33 \pm 0.06 \mathrm{eV}$ result when epitaxial $\mathrm{NiAl}$ is grown on mid-1017 $\mathrm{cm}^{-3}$ n-type and p-type buffer layers, respectively. ${ }^{17}$ Somewhat lower values $(\sim 0.9 \mathrm{eV})$ are measured on n-type substrates with no buffer layer. ${ }^{17}$ These results, coupled with the presence of interface chemistry and the associated creation of defect states, strongly suggests that NiAl pins the Fermi level deep in the forbidden gap at an energy of $\sim 0.3-0.4 \mathrm{eV}$ above the valence band maximum. According to the Schottky model, the SBH should be given by the difference between the metal work function and the semiconductor electron affinity. $20 \mathrm{It}$ is difficult to conceive of the Schottky model being obeyed by this system. A density of defects that is comparable to the $\mathrm{GaAs}(001)$ surface atom density is created upon interface formation, as betrayed by the extent of interface chemistry discussed in the previous paragraph. Such defects are expected to give rise to interface electronic states that should pin the Fermi level somewhere in the gap. As suggested by Mailhiot and Duke, the exact pinning position probably depends critically on the chemistry of interface formation, rather than assuming some universal position dicta.eul by native defects in the substrate. ${ }^{21}$ However, it is not possible to reject the Schottky model outright, in that the absolute work function of $\mathrm{NiAl}(001)$ is not known. Regardless of the physical cause of the 0.9-1.0 eV SBH at the NiAl/n-GaAs(001) interface, this high value indicates that the use of NiAl without an interlayer may result in a significant improvement in reducing gate leakage in FET applications. Any further increase in the SBH that can be achieved by means of a wide bandgap interlayer will further reduce gate leakage. In addition, it is of interest to find a small bandgap interlayer that will, in 
conjunction with $\mathrm{NiAl}$, permit reduction of the $\mathrm{SBH}$ to a value well below the midpoint energy of $0.7-0.8 \mathrm{eV}$.

\section{The use of AlAs interlayers to increase the SBH}

AlAs is an excellent choice for an interlayer to enable increasing the $\mathrm{NiAl} / \mathrm{GaAs}$ $\mathrm{SBH}$ above $1 \mathrm{eV}$ for several reasons. First, it is lattice matched to GaAs. Therefore, epitaxy of NiAl on AlAs should be straightforward, as it is on GaAs. Second, the material is more refractory than GaAs, and the AlAs/GaAs interface is stable at temperatures of several hundred degrees Celcius. As a result, overgrowth of $\mathrm{NiAl}$ at $\sim 100^{\circ}-300^{\circ} \mathrm{C}$ and subsequent operation at channel temperatures of $100^{\circ}-150^{\circ} \mathrm{C}$ should not degrade the interface. Third, the bandgap of AlAs at the $\Gamma$ point is $2.16 \mathrm{eV}$, and the AlAs/GaAs

conduction band offset is $\sim 0.34 \pm 0.07 \in V .22-24$ Thus, the SHB should be enhanced by $\sim 0.3 \mathrm{eV}$ from the value resulting from the natural band bending at the $\mathrm{NiAl} / \mathrm{GaAs}$ interface, provided the presince of the AlAs interlayer does not flatten the bands.

We show in Figure $3 \mathrm{Ga} 3 \mathrm{~d}$ core-level spectra for clean, As-decapped n-GaAs(001) with a buffer layer grown in the Perkin Elmer MBE system, for the substrate after growth of 10 monolaj'ers (ML) of AlAs in the MBE/XPS/XPD/LEED system, and after the growth of $15 \mathrm{ML}$ of epitaxial $\mathrm{NiAl}$ in the same system. ${ }^{22}$ Also shown are the energy differences between the GaAs conduction band minimum and the Fermi level in the near-surface region ( $\triangle \mathrm{E}_{\mathrm{CB}-\mathrm{FL}}$ ), as determined from the absolute $\mathrm{Ga} 3 \mathrm{~d}$ binding energies. The value of $\Delta \mathrm{E}_{\mathrm{CB}}$. FL is directly related to the amount of band bending on the GaAs side of the heterojunction. The band bending for the clean surface is such that the Fermi level is approximately at midgap, and the band bending is reduced only slightly by growth of the AlAs interlayer. $\triangle E_{C B}-F L$ increases to $0.83 \mathrm{eV}$ after growth of the $\mathrm{NiAl}$ at $260^{\circ} \mathrm{C}$ and then further increases to $\sim 0.9 \mathrm{eV}$ after annealing at $570^{\circ} \mathrm{C}$. Thus, the GaAs interface Fermi energy for the $\mathrm{NiAl} / \mathrm{AlAs} / \mathrm{n}-\mathrm{GaAs}(001)$ system is within experimental error of the value observed for $\mathrm{NiAl} / \mathrm{n}-\mathrm{GaAs}(001)$, indicating Fermi-level pinning rather deep in the forbidden gap on both AlAs and GaAs. Combining this value of $\triangle \mathrm{E}_{\mathrm{CB}} \mathrm{FL}$ with $\Delta \mathrm{E}_{\mathrm{C}}$ for the $\mathrm{AlAs} / \mathrm{GaAs}$ interface, 
the $S B H\left(\Phi_{\mathrm{B}}\right)$ becomes $\sim 1.2 \pm 0.1 \mathrm{eV}$. This value is in good agreement with the results of internal photoemission spectroscopy (IPES) measurements by Cheeks et al., which generated a value of $\sim 1.1 \mathrm{eV}$ for comparable AlAs interlayer thicknesses. ${ }^{25}$ A key physical difference between the two measurement techniques is that IPES depends on electron transport across the interface at the conduction band energy, whereas $\mathrm{Ga} 3 \mathrm{~d}$ photoelectrons generated in the XPS experiment cross the interface at energies of $\sim 1.5 \mathrm{keV}$ above the Fermi level. Therefore, IPES photoelectrons will tunnel through a thin AlAs interlayer via the X-band, which lies 0.1-0.2 eV below the AlAs conduction band minimum. In contrast, XPS photoelectrons leave the solid at an energy that is far too large to permit such tunneling. Therefore, the total SBH probed by IPES will be lower than that determined by XPS by $\sim 0.1-0.2 \mathrm{eV}$. With this difference taken into consideration, the results from XPS and IPES are in very good agreement.

The fact that the SBH is stable with respect to annealing at $570^{\circ} \mathrm{C}$ (bottom spectrum in Figure 3) reveals that the interfacial system should be able to operate at elevated channel temperarures of $100^{\circ}-150^{\circ} \mathrm{C}$ without any serious degradation. Channel temperatures in this range may result due to power dissipation beneath the gate when the FET is conducting.

\section{The use of $\mathrm{GaSe}_{\mathbf{x}} \mathrm{As}_{1-\mathrm{x}}$ interlayers for a low SBH}

One approach to achieving a low SBH by means of a small bandgap interlayer is to use a GaAs-based ternary in which the third element is an n-type dopant in GaAs. Such a material may exhibit band tailing, in which the conduction band minimum drops to the donor level in the limit of a very high extent of dopant incorporation. An obvious choice for the third element from the point of view of lattice matching is Se. The covalent radius of Se differs from that of As by only $0.04 \AA$. Therefore, the incorporation of Se into the $\mathrm{GaAs}$ lattice should preserve the GaAs lattice dimensions to such an extent that $\mathrm{NiAl}$ can be grown epitaxially as easily as on GaAs. Furthermore, band tailing might be expected to pin the Fermi level at or near the conduction band minimum, leading to a considerable reduction in band bending. We have explored these possibilities by annealing a Se-doped 
$\mathrm{GaAs}(001)$ buffer layer in $\mathrm{H}_{2} \mathrm{Se}$ at $450^{\circ} \mathrm{C}$ immediately after growth in an MOCVD reactor. We have then determined the band bending and near-surface structure with XPS, XPD, and LEED. 26

We show in Figure $4 \mathrm{Ga}, \mathrm{As}$, and $\mathrm{Se} 3 \mathrm{~d}$ azimuthal angular distributions at a polar angle $(\theta)$ of $35^{\circ}$ off the surface plane for the Se-passivated system. This surface exhibits a clear, sharp (2x1) LEED pattern, indicating that there are no missing dimers present in the surface layer. The diffraction modulation of the Se $3 \mathrm{~d}$ peak intensity is very similar to that of the As $3 d$ intensity. Furthermore, both anion angle scans are qualitatively different than that associated with $\mathrm{Ga} 3 \mathrm{~d}$ photoemission. This result establishes that Se displaces As atoms and occupies anion lattice sites. Similar scans at a variety of polar angles, in addition to polar scans, lead to the same conclusion, and allow the approximate stoichiometry of the near-surface region to be determirei. This analysis reveais that a graded ternary of $\mathrm{GaSe}_{\mathrm{x}} \mathrm{As}_{1-\mathrm{x}}$ forms in which $\mathrm{x}$ is $\sim 0.75$ in the surface anion layer and $\mathrm{x}$ is $\sim 0.25$ in the fourth anion layer in from the surface. Moreover, there is no evidence of strain in the nearsurface region.

$\mathrm{Ga}$ and $\mathrm{As} 3 \mathrm{~d}$ core-level XPS spectra for the $\mathrm{GaSe}_{\mathrm{x}} \mathrm{As}_{1-\mathrm{x}} / \mathrm{GaAs}(001)$ system are shown at the top of Figure 5, along with the SBH values determined by XPS. ${ }^{19} \Phi_{\mathrm{B}}$ is markedly reduced from the $\sim 0.7 \mathrm{eV}$ value measured for clean, MBE-grown, As-stabilized GaAs(001)-(2x4), indicating a substantial reduction in band bending. Together with the XPD results discussed in the previous paragraph, these results suggest that band flattening occurs in response to some combination of the following mechanisms: (i) Se delta doping such that the Fermi level is pinned near the conduction band minimum, (ii) elimination of missing dimers in the surface layer, which may create states deep in the gap, and (iii) formation of an overlayer of sufficiently high structural quality that dangling bends at the $\mathrm{GaSe}_{\mathrm{x}} \mathrm{As}_{1-x} / \mathrm{GaAs}(001)$ interface are satisfied. ${ }^{26}$

Also shown in Figure 5 are core-level spectra and SBH values for this surface after overgrowth of epitaxial layers of $\mathrm{Ni}, \mathrm{Al}, \mathrm{NiAl},{ }^{19}$ Reaction of the surface with $\mathrm{Ni}$ is 
substantial, as evidenced by the at tional spin-orbit pairs required to obtain a good fit to the raw spectra. However, LEED and state-specific XPD investigations for analogous growths on $\mathrm{GaAs}(001)$ reveal that an ordered compound of $\mathrm{Ni} \sim 0.5 \mathrm{Ga} \sim 0.3 \mathrm{As} \sim 0.2$ with a $\mathrm{CsCl}$ structure forms. ${ }^{27}$ In contrast, reaction with $\mathrm{Al}$ occurs to a much lesser extent. The primary interaction is Al-Ga exchange, leaving some free $\mathrm{Ga}$ on the surface. Ga metal typically exhibits a $\mathrm{Ga} 3 \mathrm{~d}$ binding energy that is $\sim 1 \mathrm{eV}$ less than that of $\mathrm{GaAs}$. However, there is no apparent reaction with As when $\mathrm{Al}$ is grown, in that the As $3 \mathrm{~d}$ line shape remains the same as that of the clean surface. LEED and XPD reveal that the Al overlayer grows as an fcc film rotated $45^{\circ}$ about the surface normal. ${ }^{28} \mathrm{Al}$ is $99 \%$ lattice matched to $\mathrm{GaAs}(001)$ when the lattices are aligned such that $(001)_{\mathrm{Al}} \|(001)_{\mathrm{GaAs}}$ and $[100]_{\mathrm{Al}} \|$ [110]GaAs.

Growth of NiAl results in an epitaxial film with the same structural quality and orientation as those grown on GaAs and AlAs(001). ${ }^{19}$ Limited interface reactivity occurs, as the XPS spectra in Figure 5 reveal. Unfortunately, the band flattening created by the formation of the $\mathrm{GaSe}_{x} \mathrm{As}_{1-\mathrm{x}} / \mathrm{GaAs}(001)$ interface is completely lost by the overgrowth of both $\mathrm{Ni}$ and $\mathrm{NiAl}$, and partially lost by the growth of the Al overlayer. $\Phi_{\mathrm{B}}$ increases to $\sim 0.9 \mathrm{eV}$ when $\mathrm{Ni}$ and $\mathrm{NiAl}$ are grown, but only to $\sim 0.5 \mathrm{eV}$ when $\mathrm{Al}$ is grown. The principal difference in interface chemistry between $\mathrm{Al}$ on the one hand, and $\mathrm{Ni}$ and $\mathrm{NiAl}$ on the other, is that overgrowth of $\mathrm{Ni}$ and $\mathrm{NiAl}$ disrupts the anion sublattice. This disruption is evidenced by both outdiffusion of Se and As, and by new peaks in the As 3d spectra shown in Figure 5. Thus, it appears that the unique interface chemistry between NiAl and $\mathrm{GaSe}_{\mathrm{x}} \mathrm{As}_{1-\mathrm{x}}(001)$ creates gap states that result in Fermi-level pinning rather deep in the gap, in a way that is similar to what accompanies NiAl growth on GaAs and AlAs(001).19 Furthermore, the process of disrupting the anion sublattice appears to be an integral step in the creation of such states. In any event, the $\mathrm{GaSe}_{\mathrm{x}} \mathrm{As}_{1-\mathrm{x}}$ interlayer, while being an excellent surface-electronic passivant for $\operatorname{GaAs}(001)$, does not appear to be useful from the point of view of lowering the NiAl/GaAs(001) SBH to a fe'w tenths of an eV. The same 
interface chemistry that causes Fermi-level pinning deep in the gap of GaAs and AlAs(001) when $\mathrm{NiAl}$ in grown appears to be operative on $\mathrm{GaSe}_{\mathrm{x}} \mathrm{As}_{1-\mathrm{x}}(001)$.

\section{E. The use of $n^{+}-$Ge and $n^{+}-S i$ interlayers to achieve a low SBH.}

It is also possible to use a heavily-doped, n-type group IV semiconductor as an interlayer for lowering the $\mathrm{SBH}$ for the same set of reasons discussed in tion IIID. Early work by Stall et al. demonstrated that an As-doped Ge interlayer and a Au metallization could be used to fabricate low contact resistance, nonalloyed Ohmic contacts to n-GaAs. ${ }^{29}$ Latter work by Grant and Waldrop extended this concept how that both high and low SBH values could be obtained by doping the Ge interlayer with p-type and ntype dopants, respectively. ${ }^{30}$ However, neither of these earlier sets of studies addressed the question of thermal stability, which is of paramount importance if this approach is to be of technological significance.

$\mathrm{Ge}$ is preferable over $\mathrm{Si}$ from the point of view of lattice matching to the substrate; the mismatch between $\mathrm{Ge}$ and GaAs is only $0.13 \%$. The epitaxy of $\mathrm{Ge}$ on $\mathrm{GaAs}(001)$ is well understood, and laminar films with a high degree of crystalline perfection can be routinely grown by MBE. ${ }^{31}$ We have reproduced earlier work by Waldrop and Grant to show that $\mathrm{SBH}$ values of $\leq 0.5 \mathrm{eV}$ can be easily achieved by doping the Ge interlayer with As and P. 31 We also have extended this work to examine thermal stability, as well as to determine the band bending when a NiAl overlayer is grown. We have found that band bending rumeins low at the $\mathrm{n}^{+}-\mathrm{Ge} / \mathrm{GaAs}$ heterojunction for 10 minute postgrowth anneal temperatures only up to $\sim 275^{\circ} \mathrm{C}$, which is slightly above the Ge growth temperature. 31 a The rise in SBH at higher temperatures is accompanied by a loss of As from the Ge layer. Similarly, epitaxial growth of $\mathrm{NiAl}$ at $\sim 200^{\circ} \mathrm{C}$ on $20 \mathrm{ML}$ films of As-doped Ge causes an immediate increase in the SBH to $\sim 0.65 \mathrm{eV}$ as metal atoms diffuse through the Ge layer and react with the GaAs substrate. ${ }^{32}$ This $\mathrm{SBH}$ is stable for 10 minute anneals at $260^{\circ} \mathrm{C}$, but increases to $0.72 \mathrm{eV}$ after an anneal at $360^{\circ} \mathrm{C}$. It is possible that the low SBH could be better preserved by using a thicker Ge interlayer. Both the indiffusion of metal atoms 
through the Ge layer and the outdiffusion of As may be impeded by increasing the interlayer thickness. However, we have not done so because XPS cannot be used to measure the SBH unless the total inter- and overlayer thickness is less than $\sim 3 \lambda \sin \theta$, where $\lambda$ is the photoelectron escape length and $\theta$ is the polar angle of detection.

Although the latice mismatch between $\mathrm{Si}$ and $\mathrm{GaAs}$ is $4 \%, \mathrm{n}^{+}-\mathrm{Si}$ interlayers show considerably more promise from the point of view of thermal stability. We show in Figure $6 \mathrm{Ga} 3 \mathrm{~d}$ and Si $2 \mathrm{p}$ core-level spectra for undoped and heavily As-doped epitaxial Si overlayers on $n-G a A s(001)-(2 \times 4) .33$ Overgrowth of undoped Si causes only a slight reduction in the SBFi relative to the clean surface. However, doping the Si layer with As causes $\triangle \mathrm{E}_{\mathrm{CB} \text {-FL }}$ to be reduced to $0.27 \mathrm{eV}$, presumably as a result of electron transfer from the overlayer into the substrate and concomitant reduction in the electric field across the depletion region. An absolute Si 2p core-level binding energy of $99.98 \mathrm{eV}$ indicates that the $\mathrm{Si}$ layer is nearly negenerately doped $\mathrm{n}$-type. Thirty minute anneals at temperatures of $450^{\circ} \mathrm{C}$ caused no measurable increase in the SBH, indicating excellent thermal stability.

Unfortunately, the $4 \%$ lattice mismatch causes lattice relaxation for coverages above the critical thickness. We have used XPD to determine the critical thickness by measuring the polar angle at which the zeroth-order, forward-scattering peak along the [101] lowindex direction occurs. 33 This peak, which falls at $45.0^{\circ}$ in unstrained $\mathrm{Si}$, is expected to come at a smaller polar angle as a result of tetragonal distortion accompanying accomodation to the substrate lattice. Indeed, an average value of $43.4 \pm 0.3^{\circ}$ is observed for thicknesses up to $\sim 10 \AA$, above which $\theta_{[101]}$ begins to monotonically increase, signaling the onset of lattice relaxation. A plot of $\theta_{[101]}$ as a function of coverage for growth at $400^{\circ} \mathrm{C}$ is shown in the bottom panel of Figure 7. In addition, the LEED bearns begin to broaden at a coverage of $12 \AA$, indicating the onset of in-plane relaxation. Lattice relaxation is accompanied by island formation in the Si layer. Island formation is also expected on the basis of the high surface free energy of Si. We have detected island formation by two methods. First, the rate of attenuation of $\mathrm{Ga} 3 \mathrm{~d}$ intensity from the substrate is consistent 
with island formation. We plot in the top panel of Figure 7 the total amount of $\mathrm{Si}$ deposited, as determined by Rutherford backscattering, against the apparent Si overlayer thickness based on $\mathrm{Ga} 3 \mathrm{~d}$ attenuation. The line with unity slope is what is expected if perfect laminar growth occurs. The positive deviation from linearity is consistent with island formation. In addition, these islands have been directly imaged by atomic force microscopy for thicker coverages. ${ }^{33}$ Therefore, it does not appear possible to grow fullylaminar, strained Si on GaAs with sufficient thickness to act as a suitable interlayer for the purpose of controlling the band bending. However, as we discuss below, $\mathrm{n}^{+}-\mathrm{Si}$ interlayers at thicknesses in excess of the critical thickness do effectively lower the SBH at the $\mathrm{NiAl} / \mathrm{n}^{+}-\mathrm{Si} / \mathrm{n}-\mathrm{GaAs}(001)$ system.

We have grown $30 \AA \mathrm{NiAl} / 50 \AA \mathrm{n}^{+}-\mathrm{Si} / \mathrm{n}-\mathrm{GaAs}(001)$ heterojunctions that exhibit $\mathrm{SBH}$ values of $0.4-0.5 \mathrm{eV}$ after growth, and after post-growth heat treatments of 30 minutes at up to $475^{\circ} \mathrm{C}$. Similarly, we have measured hole SBH values of $0.8-0.9 \mathrm{eV}$ for the same layer structure grown on $\mathrm{p}-\mathrm{GaAs}(001)$. The NiAl overlayers grown to date have been poorly-ordered single crystals, presumably because the growth template is nearly fully-relaxed $\mathrm{Si}$ to which $\mathrm{NiAl}$ is not well lattice matched. However, the excellent thermal stability suggests that this particular system may be useful in device technology, as explained in the next section.

\section{F. Application -- complementary inverter with low leakage current.}

In order to fabricate a complementary inverter with low leakage current, it is required that the $\mathrm{SBH}$ on both $\mathrm{n}$ - and $\mathrm{p}$-channels be sufficiently high that gate leakage is negligible when the two channels are populated. Threshold voltages (defined as the gate voltage required to initiate carrier population in the channel) of $+0.3 \mathrm{eV}$ and $-0.3 \mathrm{eV}$ for the $\mathrm{n}$ - and p-channels, respectively, can be achieved by selective channel doping. Using these threshold voltages, full channel turn on should be attainable with gate voltages of $\sim \pm 0.6$ $0.7 \mathrm{eV}$ (+ for $\mathrm{n}$-channel, - for $\mathrm{p}$-channel). However, gate leakage will occur if the SBH is less than the voltage required for full channel turn on. Thermally-stable SBH values of 
$0.9 \mathrm{eV}$ now appear achievable on $\mathrm{n}$ - and $\mathrm{p}-\mathrm{GaAs}$ by means of NiAl gate metal directly on $\mathrm{n}$-type material and an $\mathrm{n}^{+}-\mathrm{Si}$ interlayer on the p-type material. This combination of gate materials could be fabricated by MBE, as shown schematically at the top of Figure 8 . The appropriate III-V epilayer structure involving $\mathrm{n}$ - and $\mathrm{p}$-layers could be grown with the $\mathrm{p}$ layer on top. The $\mathrm{n}^{+}-\mathrm{Si}$ layer could then be grown in situ, followed by the blanket MBE growth of NiAl. Thus, the entire channel and gate structure for the p-channel devices would be prepared in ultrahigh vacuum conditions by MBE. After fabrication of the pchannel devices, the surrounding epistructure could be etched back to expose the n-layer. $\mathrm{NiAl}$ could then be grown in a standard metallization chamber by growing alternating layers of $\mathrm{Ni}$ and $\mathrm{Al}$, followed by ex-situ rapid thermal annealing to form the intermetallic. The $\mathrm{NiAl} / \mathrm{GaAs}(001)$ interface prepared in this fashion also exhibits an $\sim 1 \mathrm{eV} \mathrm{SBH} .{ }^{34}$ The $\mathrm{n}$ channel devices could then be fabricated to complete the process.

The $\sim 0.9 \mathrm{eV} \mathrm{SBH}$ on both $\mathrm{n}$ - and p-channels would significantly reduce gate leakage compared to conventional gate metallization schemes that pin the Fermi level near midgap. The energy diagrams in the bottom half of Figure 8 depict the $\mathrm{n}$ - and $\mathrm{p}$-channels with no gate voltage applied (at left), and with a gate voltage sufficient to populate each channel (at right). The high SBH values prevent carriers in the channels from tunneling through the Schottky barrier and out the gate metallization. Thus, current will be drawn from a coupled pair of $\mathrm{n}$ - and p-channel FETs only during switching, and power consumption will be minimized.

\section{Conclusions}

We have demonstrated that control over the $\mathrm{SBH}$ at metal/GaAs interfaces can be gained through the judicious use of variable-bandgap interlayers, along with epitaxial NiAl as the metal of choice. NiAl creates a $0.9-1.0 \mathrm{eV} \mathrm{SBH}$ on $\mathrm{n}-\mathrm{GaAs}(001)$ without any interlayer, and $1.2 \mathrm{eV}$ can be achieved when an AlAs interlayer is used. Interlayers of $\mathrm{n}^{+}$ Si significantly flatten the bands on $\mathrm{n}-\mathrm{GaAs}$, and a $\mathrm{SBH}$ of $0.4-0.5 \mathrm{eV}$ can be attained when $\mathrm{NiAl}$ metal is grown on the $\mathrm{Si}$ interlayer. Growth of $\mathrm{NiAl} / \mathrm{n}^{+}-\mathrm{Si} / \mathrm{p}-\mathrm{GaAs}(001)$ 
structures results in hole barrier heights of $0.8-0.9 \mathrm{eV}$. The thermal stability of these interfaces is excellent, with no thermal degradation of the SBH for anneals at temperatures up to $475^{\circ} \mathrm{C}$. The use of epitaxial $\mathrm{NiAl}$ as a gate metal with $\mathrm{n}^{+}-\mathrm{Si}$ interlayers is proposed as a way to fabricate low-leakage complementary inverters.

Acknowledgements -- The author gratefully acknowledges the skillful contributions of V.A. Loebs, V.S. Sundaram, D.H. Doyle, R. Friedman, and J.M. Cech to this work. 


\section{References}

1. S.M. Sze, 2nd ed., Physics of Semicondcutor Devices (Wiley-Interscience, New York, 1981), Chap. 5.

2. A. Piotrowska, A. Guivarch and G. Pelous, Solid-State Electronics 26179 (1983) and references therein.

3. M. Murakami, K.D. Childs, J.M. Baker and A. Callegari, J. Vac. Sci. Technol. B4, 903 (1986).

4. S. Fujita and T. Mizutani, IEEE Trans. Elec. Dev. 34, 1889 (1987).

5. L.J. Brillson, Surf. Sci. Rep. 2, 123 (1982) and references therein.

6. G. Margaritondo, Solid State Electron. 26, 499 (1983).

7. W.E. Spicer and W.E. Eglash, in VLSI Electronics: Microstructure Science (Academic Press, New York, 1985), Vol. 10, p. 79.

8. W. E. Spicer, Z. Liliental-Weber, E. Weber, N. Newman, T. Kendelewicz, R. Cao, C. McCants, P. Mahowald, K. Miyano, and I. Lindau, J. Vac. Sci. Technol. B6, 1245 (1988).

9. W.E. Spicer, P.W. Chye, P.R. Skeath, C.Y. Su, and I. Lindau, J. Vac. Sci. Technol. 16, 1427 (1979); 17, 1019 (1980); Phys. Rev. Lett. 44, 420 (1980).

10. K. Ludeke, G. Jezequel, and A. Taleb-Ibrahimi, J. Vac. Sci. Technol. B6, 1277 (1988) and references therein.

11. L.J. Brillson, Phys. Rev. Lett. 40, 260 (1978); Phys. Rev. 18, 2431 (1978).

12. J. L. Freeouf and J. M. Woodall, Appl. Phys. Lett. 39, 727 (1981).

13. J. Tersoff, Phys. Rev. Lett. 52, 465 (1984); Phys. Rev. B30, 7874 (1984).

14. V. Heine, Phys. Rev. A138, 1689 (1965); C. Tejedor, F. Flores, and E. Louis, J. Phys. C 10, 2163 (1977).

15. T. Sands, C.J. Palmstr $\phi$ m, J.P. Harbison, V.G. Keramidas, N. Tabatabaie, T.L. Cheeks, R. Ramesh and Y. Siberberg, Mat. Sci. Rep. 5, 99 (1990) and references therein. 
16. S.A.Chambers, J. Vac. Sci. Technol. A8, 2062 (1990), and, Phys. Rev. B42, 10865 (1990).

17. S.A. Chambers and V.A. Loebs, J. Vac. Sci. Technol. B8, 724 (1990), and J. Vac.

Sci. Technol. A, to appear, May-June issue (1992).

18. S.A. Chambers, J. Vac. Sci. Technol. B7, 737 (1989).

19. S.A. Chambers and V.A. Loebs, Mat. Res. Soc. Proc. 221, 283 (1991).

20. W. Schottky, Naturwissenschaften 26, 843 (1938).

21. C.B. Duke and C. Mailhiot, J. Vac. Sci. Technol. B3, 1170 (1985).

22. S.A. Chambers, V.A. Loebs and D.H.Doyle, J. Vac. Sci. Technol. B8, 985 (1990).

23. J.R. Waldrop, R.W. Grant, and E.A. Kraut, J. Vac. Sci. Technol. B5, 1209 (1987).

24. E.T. Yu, D.H. Chow, and T.C. McGill, J. Vac. Sci. Technol. B7, 391 (1989).

25. T.L. Cheeks, T. Sands, R.E. Nahory, J. Harbison, N. Tabatabaie, H.L. Gilchrist, B.J. Wilkens, and V.G. Keramidas, Appl. Phys. Lett. 56, 1043 (1990).

26. S.A. Chambers and V.S. Sundaram, Appl. Phys. Lett. 57, 2342 (1990), and, J. Vac. Sci. Technol. B9, 2256 (1991).

27. S.A. Chambers and V.A. Loebs, Appl. Phys. Lett. 60, 38 (1992).

28. S.A. Chambers, Phys. Rev. B39, 12664 (1989).

29. R.A. Stall, C.E.C. Wood, K. Board, N. Dandekar, L.F. Eastman, and J. Devlin, J. Appl. Phys. 52, 4062 (1981).

30. R.W. Grant and J.R. Waldrop, J. Vac. Sci. Technol. B5, 1015 (1987), and, Appl. Phys. Lett. 52, 1794 (1988).

31. S.A. Chambers and T.J. Irwin, Phys. Rev. B38, 7484 (1988), and, Phys. Rev. B38, 7858 (1988).

32. S.A. Chambers and V.A. Loebs, J. Vac. Sci. Technol. A8, 2074 (1990).

33. S.A. Chambers and V.A. Loebs, Phys. Rev. B, submitted (1992).

34. T. Sands, W.K. Chan, C.C. Chang, E.W. Chase and V.G Keramidas, Appl. Phys. Lett. 52, 1338 (1988). 


\section{Eigure Captions}

Fig. 1 Schematic energy diagrams illustrating the effect of variable band gap interlayers in tailoring the Schottky barrier height at a metal/semiconductor interface.

Fig. 2 (Top) Ga and As $3 \mathrm{~d}$ reduced intensities as a function of NiAl overlayer coverage. (Bottom) Number of reacted GaAs bilayers vs. coverage, as determined by modeling the ratio of reacted to substrate $\mathrm{Ga} 3 \mathrm{~d}$ intensities. The growth temperature was $100 \mathrm{C}$. Taken from ref. $17 \mathrm{~b}$.

Fig. $3 \mathrm{Ga} 3 \mathrm{~d}$ core-level spectra obtained at normal emission $\left(\theta=90^{\circ}\right)$, along with the energy difference between the conduction band minimum and the Fermi-level $\left(\triangle \mathrm{E}_{\mathrm{CB}-\mathrm{FL}}\right)$, for the NiAl/AlAs/GaAs(001) heterojunction, Taken from ref. 22.

Fig. $4 \mathrm{Ga}$, As and Se 3d azimuthal angle intensicy distributions at a polar angle $(\theta)$ of $35^{\circ}$ off the surface plane for Se-passivated $\mathrm{GaAs}(001)$. Taken from ref. 26a.

Fig. $5 \mathrm{Ga}$ and As $3 \mathrm{~d}$ core-level spectra, along with SBH values, for metal overlayers on Se-passivated GaAs(001). Taken from ref. 19.

Fig. $6 \mathrm{Ga} 3 \mathrm{~d}$ and $\mathrm{Si} 2 \mathrm{p}$ core-level spectra and $\Delta \mathrm{E}_{\mathrm{CB}-\mathrm{FL}}$ values for $\mathrm{Si}$ overlayers on $\mathrm{n}$ GaAs(001), taken from ref. 33 .

Fig. 7 (Top) Total Si deposit, as judged by Rutherford backscattering, vs. apparent Si overlayer thickness from $\mathrm{Ga} 3 \mathrm{~d}$ attenuation for Si overlayers on $\mathrm{GaAs}(001)$. (Bottom) Polar angle of the [101] zeroth-order Si 2p x-ray photoelectron diffraction peak vs. overlayer thickness for Si on $\mathrm{GaAs}(001)$. Taken from ref. 33.

Fig. 8 Device structure (top) and energy diagrams (bottom) for a low-leakage complementary inverter in which epitaxial NiAl is used as the gate metal, and an $\mathrm{n}^{+}$Si interlayer is used in the p-channel. 


\section{HIGH SCHOTTKY BARRIER HEIGHT}
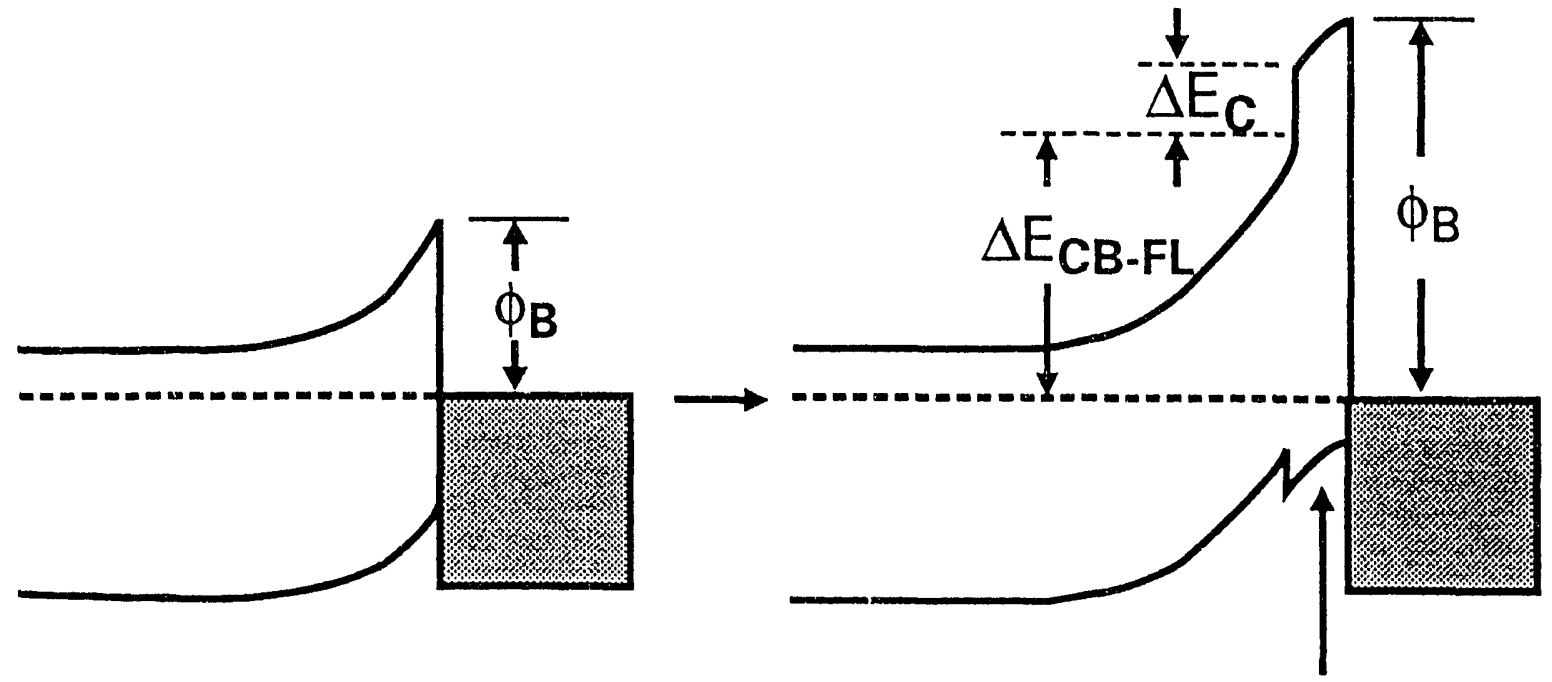

interlayer

\section{LOW SCHOTTKY BARRIER HEIGHT}
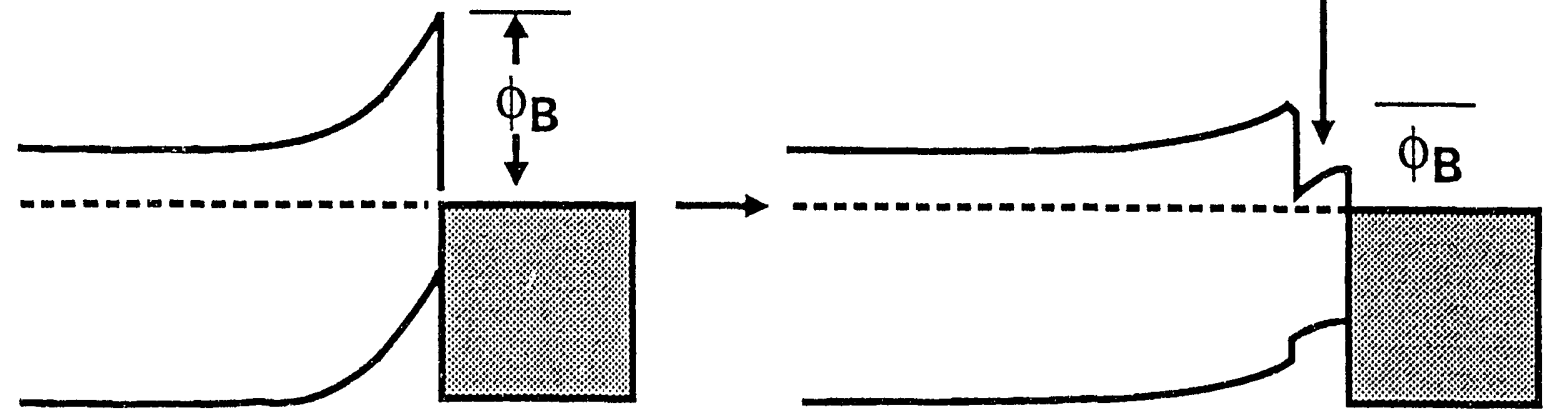
NiAI OVERLAYER THICKNESS $(\AA)$
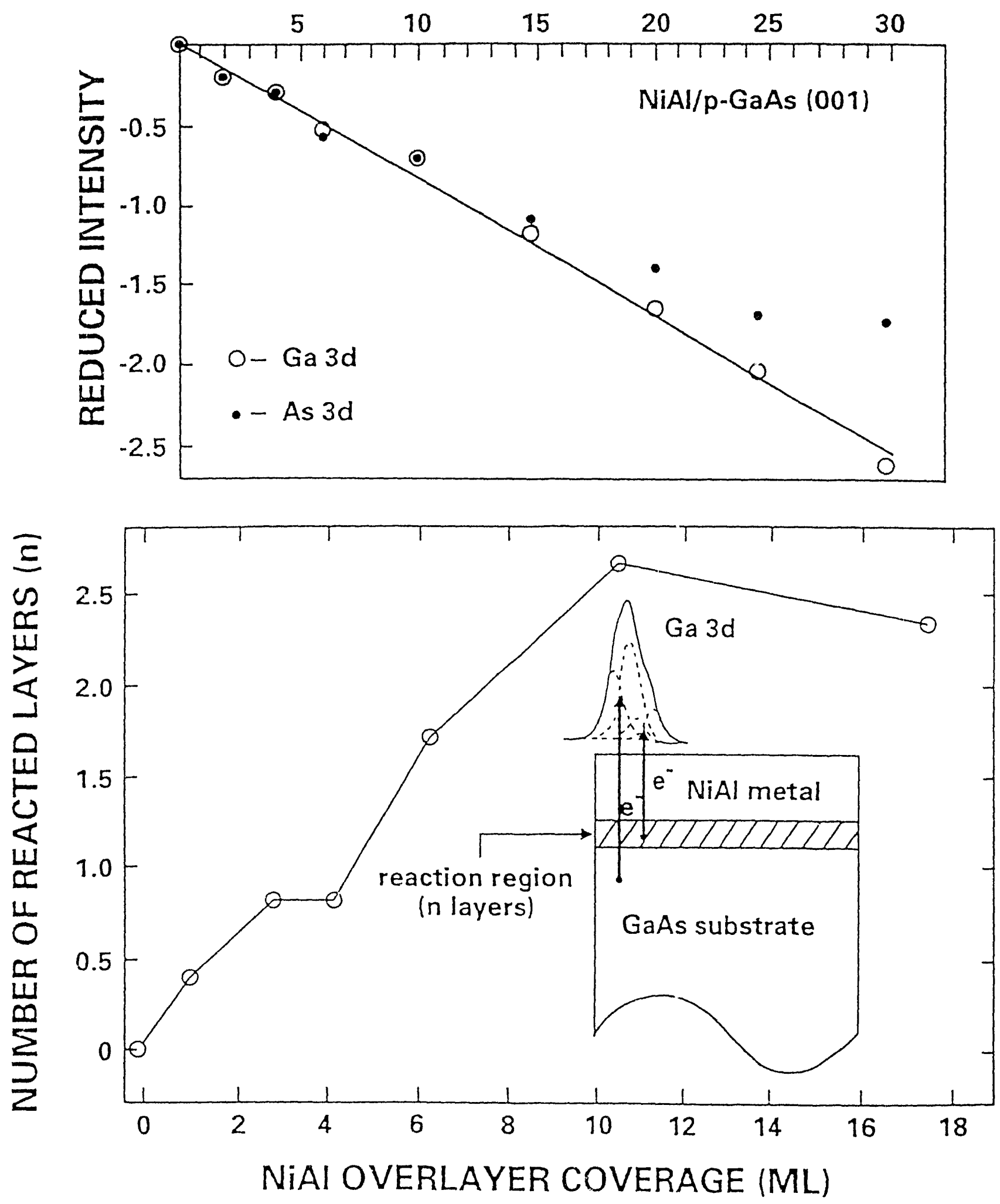


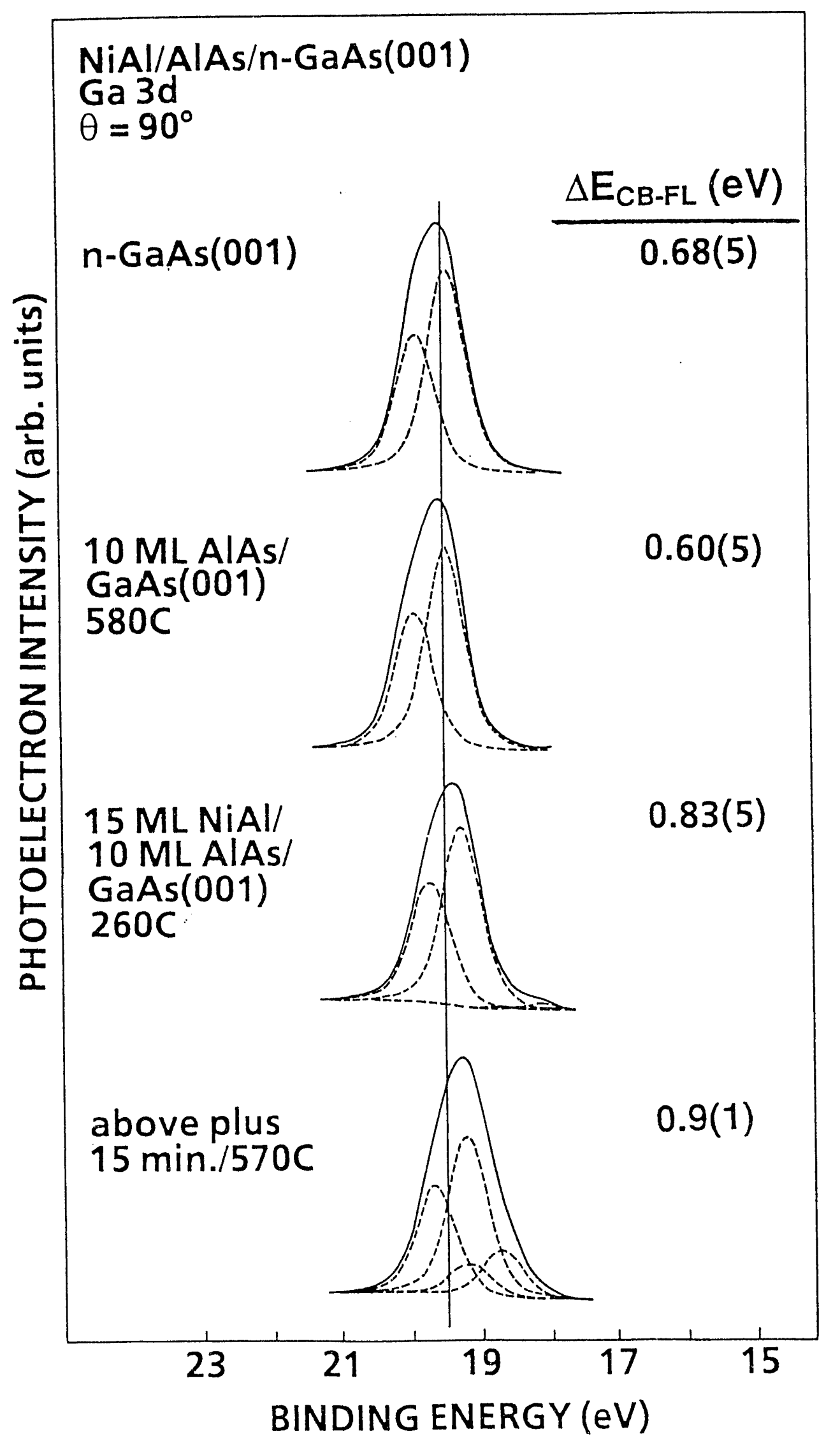

Figure 3 


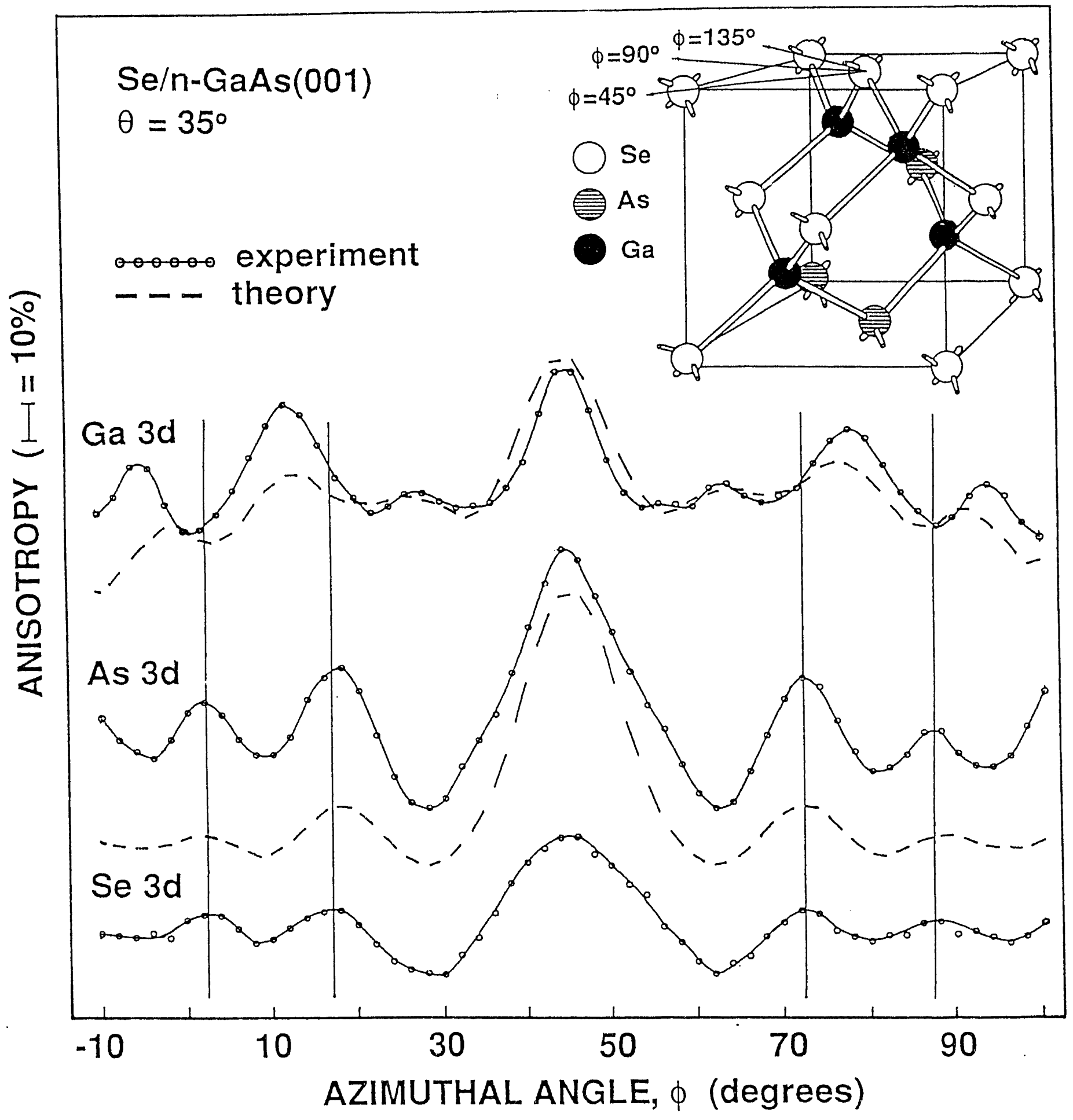

Figure 4 


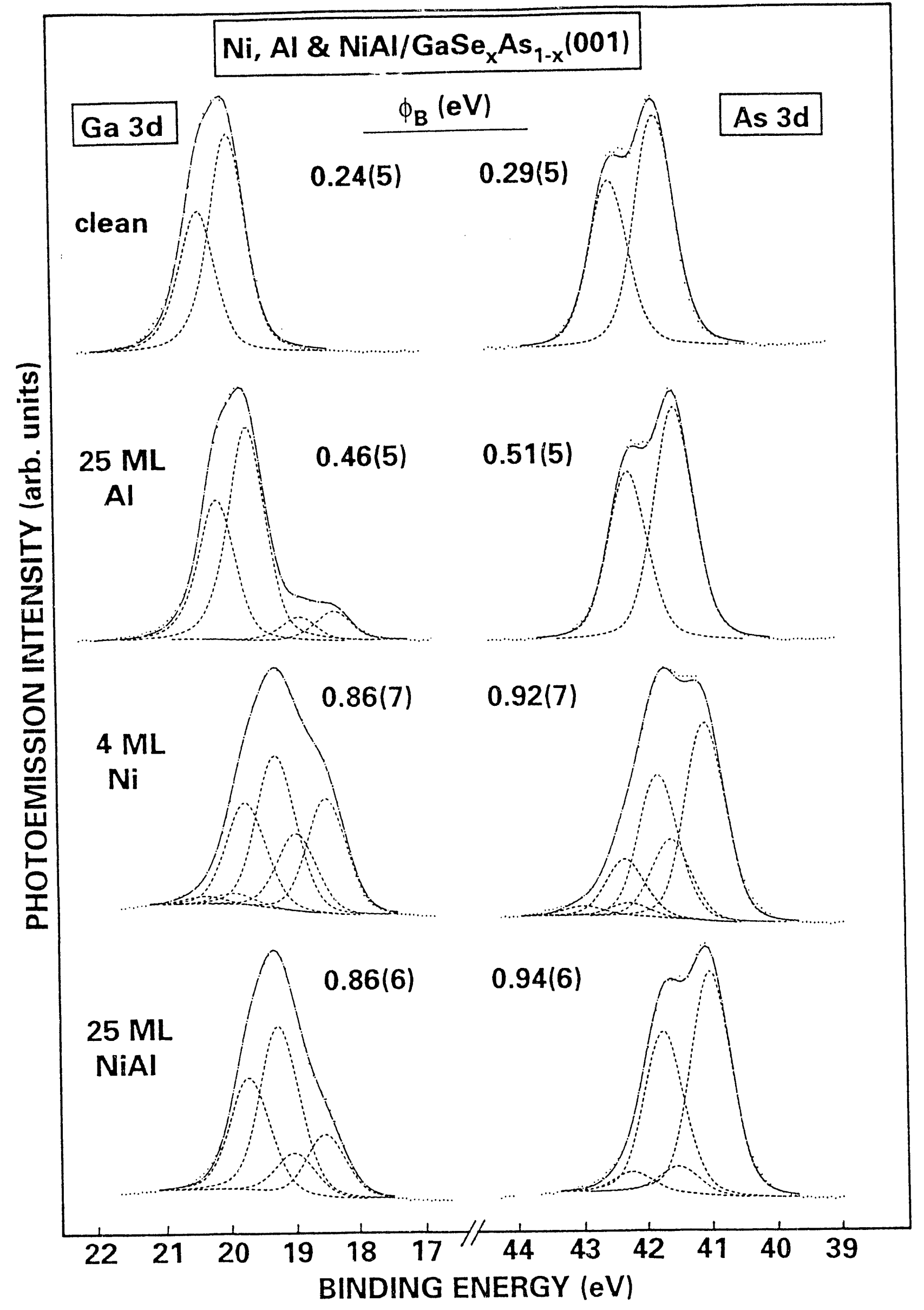

Figure 5 


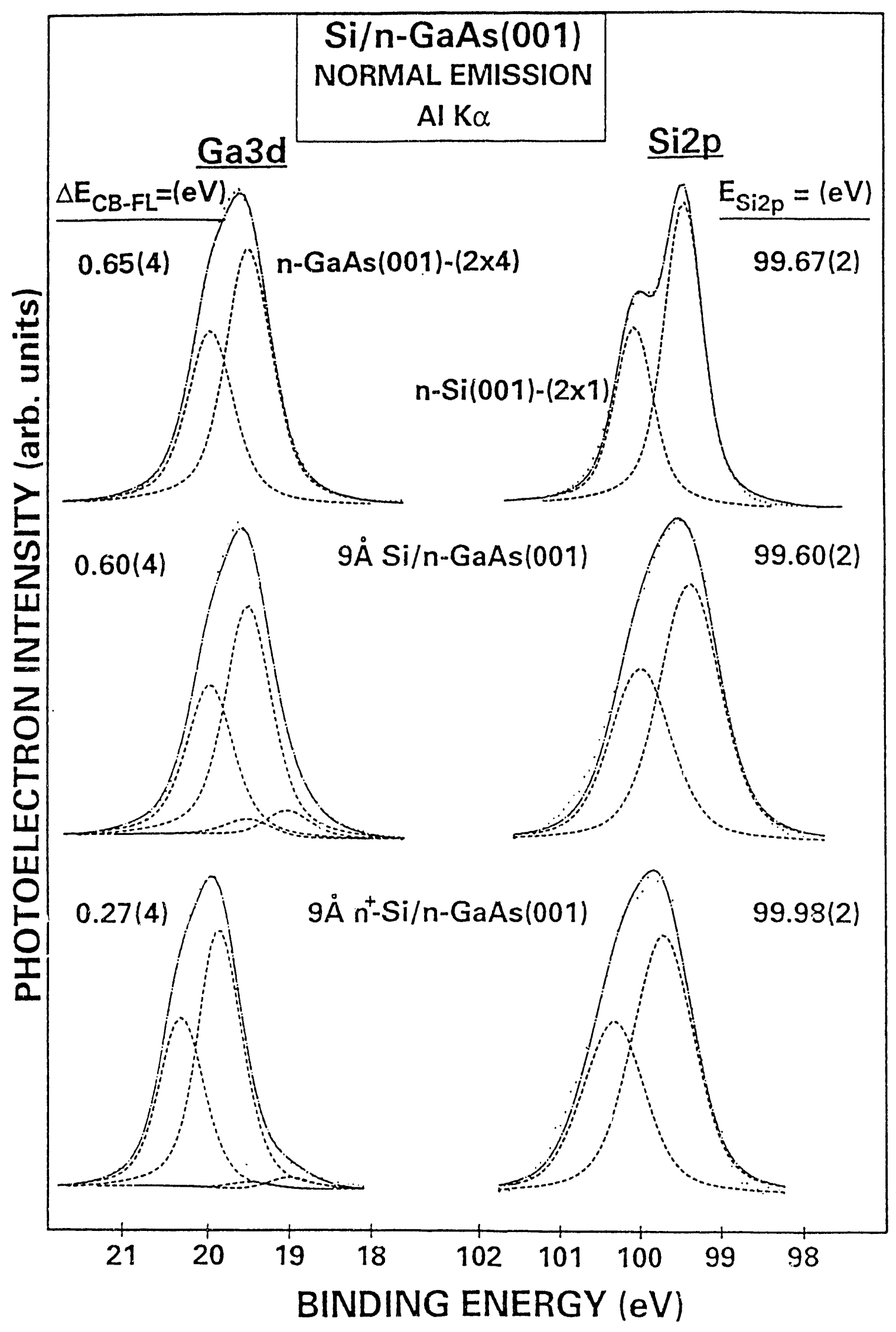

Figure 6 


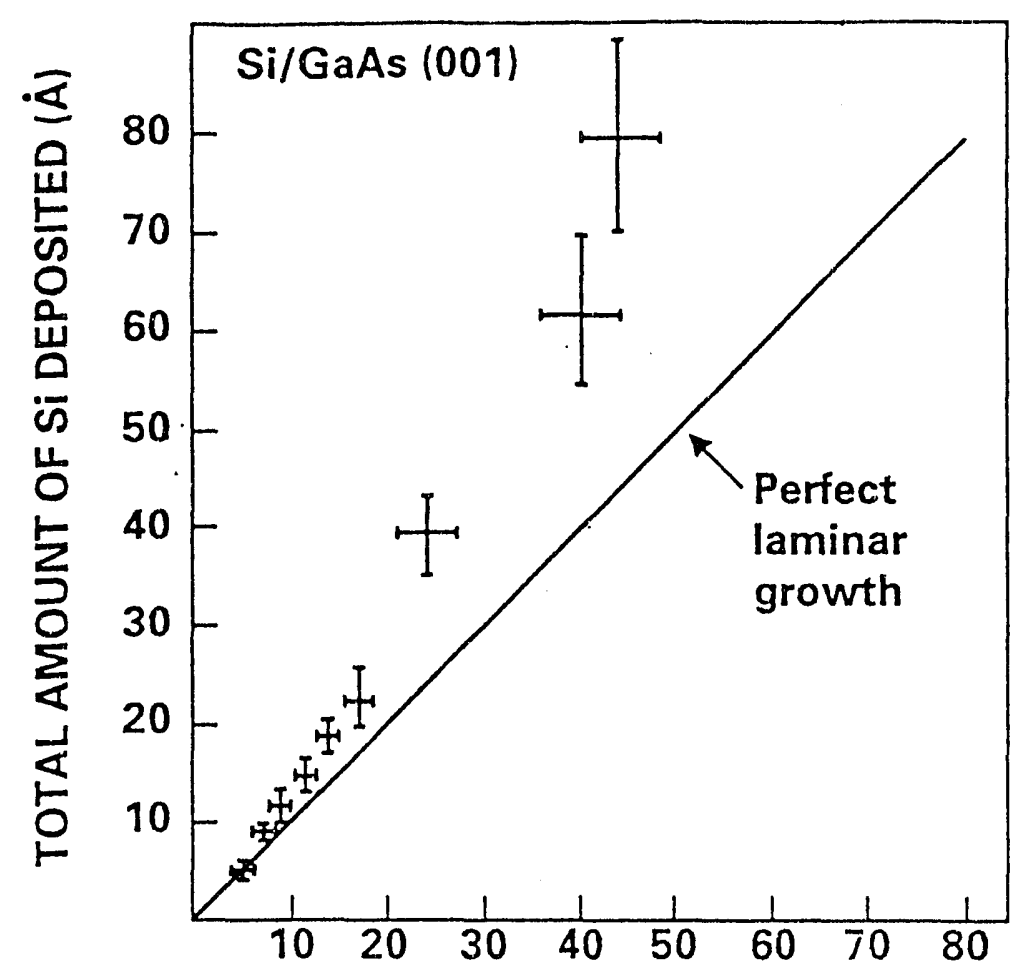

Si OVERLAYER THICKNESS BASED ON Ga 3d ATTENUATION $(\AA)$

Si OVERLAYER THICKNESS (ML)

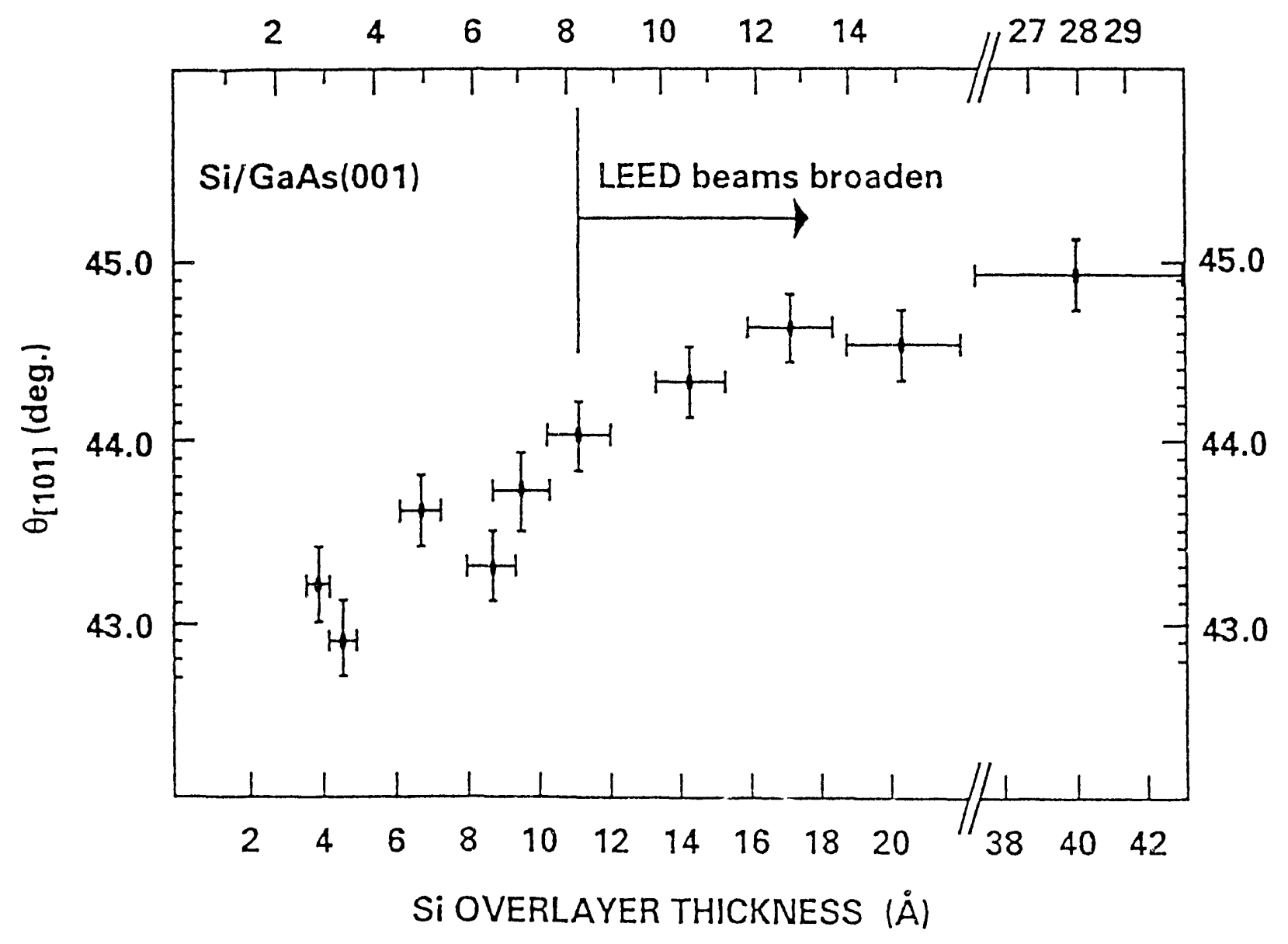




\section{COMPLEMENTARY INVERTER WITH LOW LEAKAGE CURRENT}

\section{n-Channel FET}

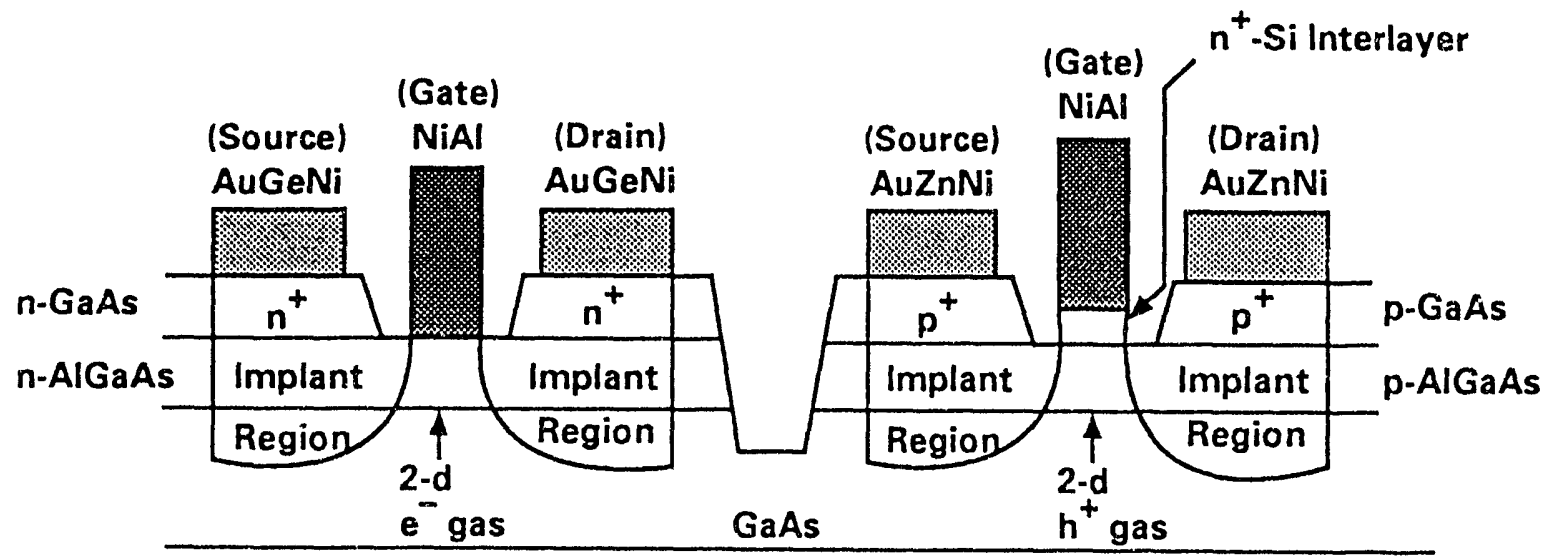

\section{n-Channel Gate}
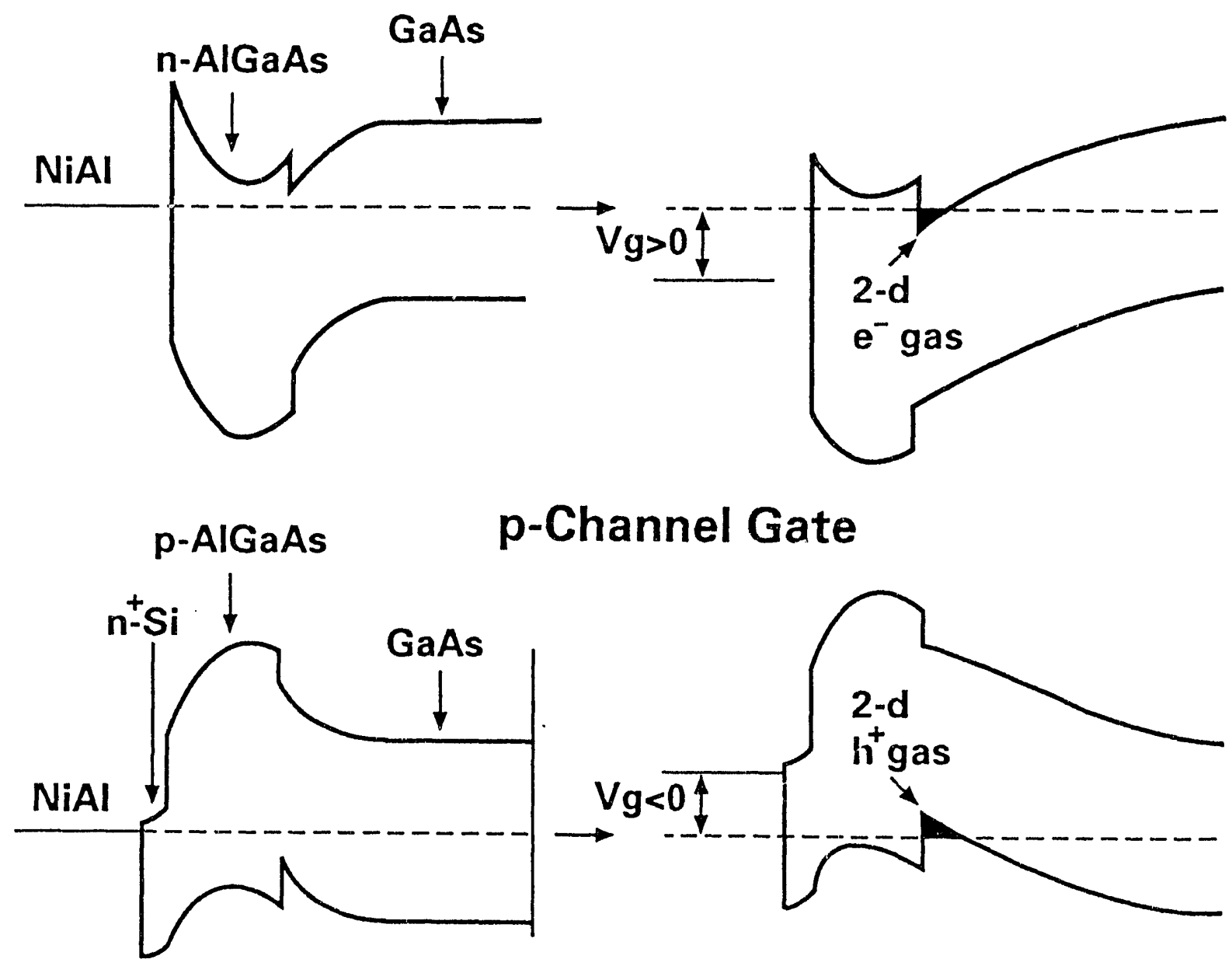

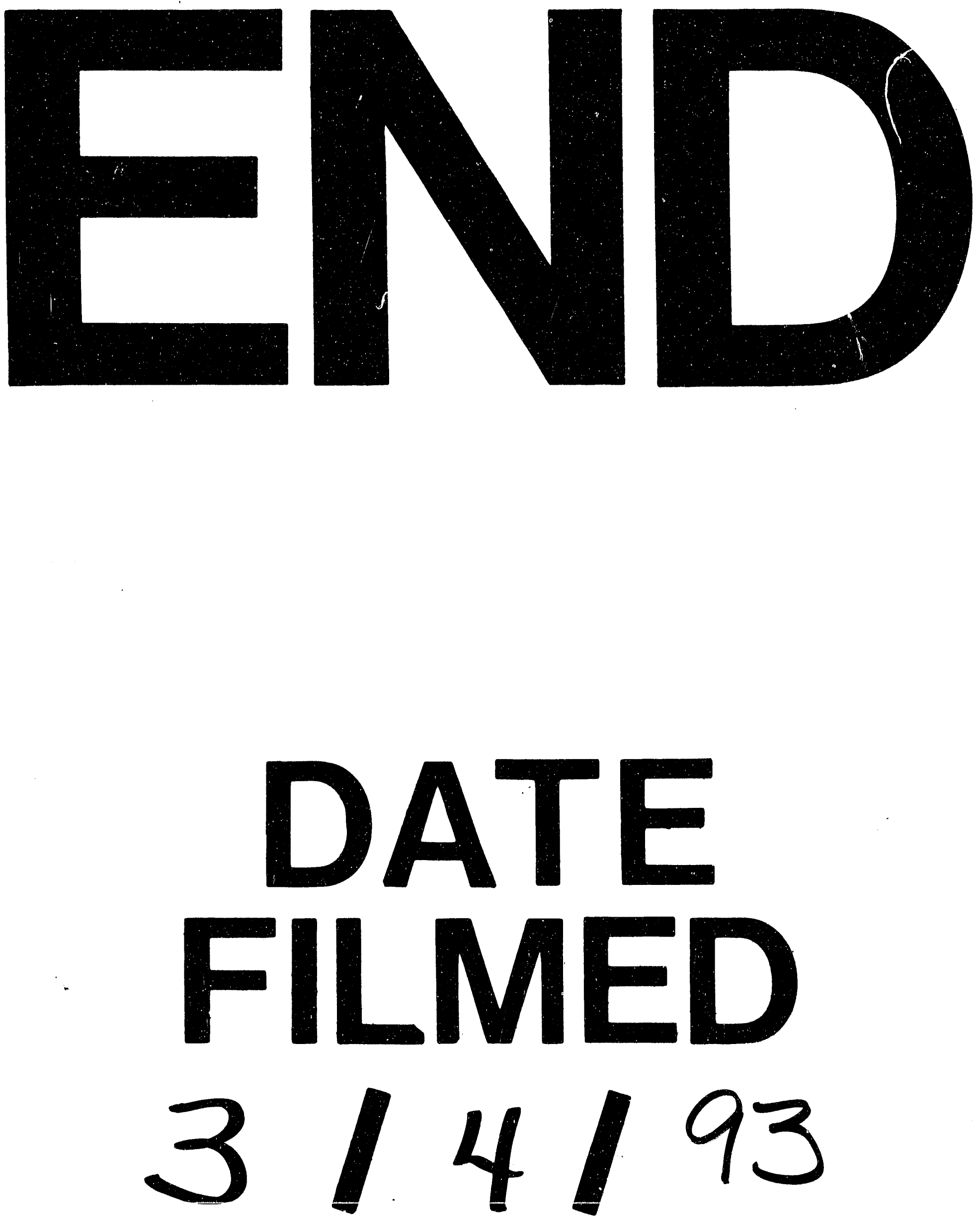
